\title{
Near-Infrared Tunable Reflection and Absorption Using Nanostructured Thin Film Structures Employing Phase-Change Material
}

\author{
A. OZDEMIR* AND H. KOCER \\ Turkish Military Academy, Department of Electrical Engineering, 06654, Ankara, Turkey
}

\begin{abstract}
We present the design of a polarization-dependent tunable nanostructured thin film absorber in the nearinfrared region. Germanium antimonide tellurite (GST) was employed as the phase change material in the designed structure. Our structure is composed of a periodic grating-type array of $150 \mathrm{~nm}$ thick Au buried with $50 \mathrm{~nm}$ thick GST layer from the top of the Au layer. The period of the gratings is $2 \mu \mathrm{m}$ and in each period, GST width is $1 \mu \mathrm{m}$. GST was selected as the active phase change material because its optical properties undergo a substantial change during a structural transition from amorphous to crystalline phase. The optical absorption and reflection properties of the designed structure with respect to the geometric and material parameters were systematically investigated using the finite difference time domain computations. It was shown that absorption peak or reflection dip at the resonant wavelengths in the near-infrared region was red shifted from $2039 \mathrm{~nm}$ to $2143 \mathrm{~nm}$ wavelength by switching the phase change material from its amorphous to crystalline states. The distributions of the electric field and absorbed power at the resonant wavelengths with respect to different phases of the GST were investigated to further explain the physical origin of the absorption. Our study provides a path toward the realization of tunable infrared absorbers for applications, such as selective infrared emitters, infrared camouflage, sensors, and photovoltaic devices.
\end{abstract}

DOI: 10.12693/APhysPolA.129.464

PACS/topics: 78.67.Pt

\section{Introduction}

Interaction of electromagnetic radiation with metamaterial and plasmonic nanostructures have attracted much attention since engineering the optical properties of these structures provides us very useful and various selective narow or broadband spectral control over different portions of the electromagnetic spectrum. Various studies were carried out to obtain spectral selective perfect or near perfect absorption over microwave [1], terahertz [2], infrared (IR) [3-5] and visible [6, 7] bands of electromagnetic spectrum. Engineering the spectral absorption and reflection properties in the IR range has several potential applications such as target recognition, biochemical sensing, camouflage, infrared signature mimicry, imaging, sensors, IR labeling and wavelength selective IR sources $[8,9]$. In this regard, having a tunable resonance response for IR wavelength range is a desired feature for thermal emitters [10], thermophotovoltaic cells [11], as well as plasmonic scatters [1214]. In order to dynamically or actively tune the absorption and reflection, certain tunable materials, which are called phase-change materials (PCMs) can be employed in the design [15]. Electrical [16], optical [17] and thermal [18] tunability were experimentally studied as the main active tuning mechanisms. Thermally tunable materials, such as niobium dioxide $\left(\mathrm{NbO}_{2}\right)$ and vanadium

\footnotetext{
* corresponding author; e-mail abdurrahmanozdemir@kho.edu.tr
}

dioxide $\left(\mathrm{VO}_{2}\right)$ are the examples of thermochromic materials, which change their optical properties as a function of temperature [19]. Another PCM is germanium antimonide tellurite (GST). Significantly different optical properties are obtained while the GST is in the amorphous and crystalline phases [20]. Additionally, it can be reliably and very rapidly (the switching time is $<500 \mathrm{ps}$ ) switched by electrical or optical pulses and it is scalable [15]. Therefore, it has been used for applications in rewritable optical data storage [21]. In this study, we selected $\mathrm{Ge}_{2} \mathrm{Sb}_{1} \mathrm{Te}_{4}$ (abbreviated as GST) as the PCM. Employing the GST in our nanostructured thin film design, we propose and investigate the tunable reflection and absorption mechanism in the near-infrared region using the phase change capability of the GST.

\section{Proposed design and simulation approach}

Schematic of the proposed design is shown in Fig. 1. Our design has thick Au coating (100 nm) as the backside reflector. IR illumination is sent from the nanostructured side. The designed structure is suspended in air. The unit structure is periodic along the $x$-axis with a $2 \mu \mathrm{m}$ periodicity. $50 \mathrm{~nm}$ thick GST is buried into $150 \mathrm{~nm}$ thick Au. The width of the GST is $1 \mu \mathrm{m}$. Transmission through device is zero since there exists optically thick Au layer at the bottom of the device, which prevents the light transmission. Au is chosen as the host metallic layer due to its stability and low ohmic loss.

The full-field electromagnetic wave simulations were performed using Lumerical, a commercially available finite-difference time-domain (FDTD) simulation software package [22]. The FDTD simulations reported in 


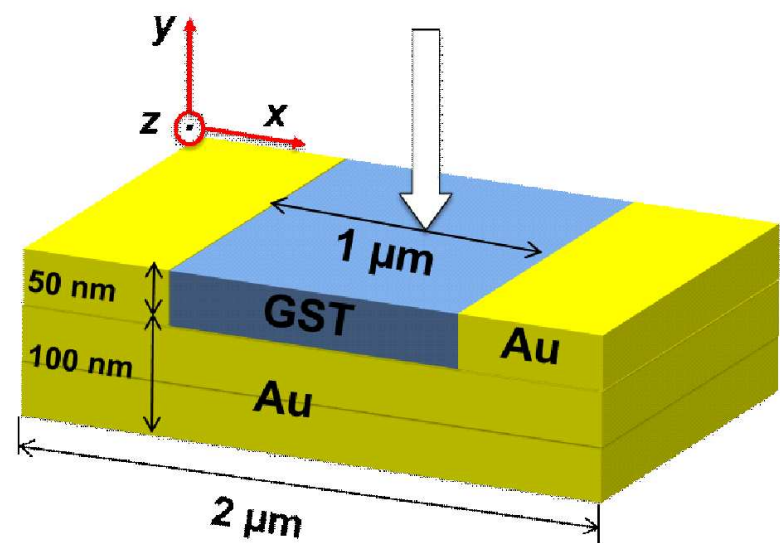

Fig. 1. Schematic of the nanostructured thin film based absorber. Plane wave is normally incident on the absorber surface. The structure is periodic along the $x$ axis with a $2 \mu \mathrm{m}$ periodicity. $50 \mathrm{~nm}$ thick GST is buried into $150 \mathrm{~nm}$ thick $\mathrm{Au}$. The width of the GST is $1 \mu \mathrm{m}$.

this paper were performed in two-dimensional layouts. In the simulations, total transmission $(T)$ and reflection $(R)$ from the structures were used to compute the absorption $(A)$ by $1-T-R$, which reduces to $1-R$ due to the optically thick $\mathrm{Au}$ back reflector. Periodic boundary conditions were used along the $x$ and $z$ axes, and perfectly matched layers were chosen along the propagation of electromagnetic waves ( $y$ axis). Plane waves were launched normally incident to the unit cell along the $-y$ direction, and reflection was monitored with a power monitor placed behind the radiation source. Electric fields and absorbed power were detected within the frequency profile and power monitor.

In the FDTD simulations, complex refractive indices for two different phases (amorphous and crystal) of GST film were taken from earlier experimental study [20]. Complex refractive index of $\mathrm{Au}$ was taken from Palik database [23]. Accordingly, wavelength dependence of real $\left(\varepsilon_{1}\right)$ and imaginary $\left(\varepsilon_{2}\right)$ parts of corresponding relative dielectric permittivities for $\mathrm{Au}$, amorphous and crystal phases of GST were plotted in Fig. 2.
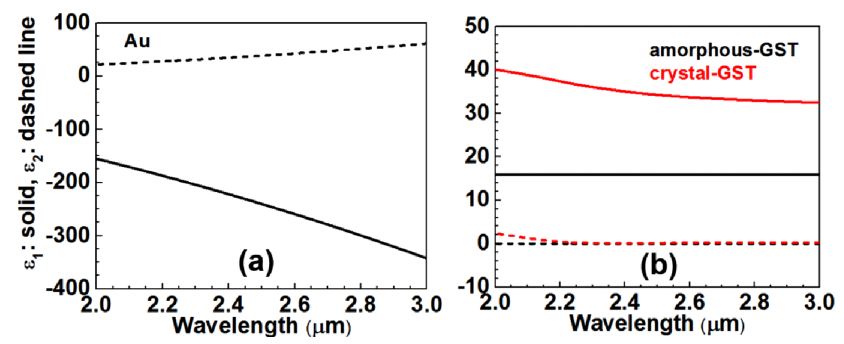

Fig. 2. Wavelength dependence of real $\left(\varepsilon_{1}\right.$, plotted in solid lines) and imaginary $\left(\varepsilon_{2}\right.$, plotted in dashed lines) parts of relative dielectric permittivities of (a) $\mathrm{Au}$ and (b) amorphous phase of GST (black-colored lines) and crystal phase of GST (red-colored lines) (Au data is from Ref. [23] and GST data is from Ref. [20]).

\section{Results and discussion}

In Fig. 3, simulation results are shown for our design. When the electric field vector is perpendicular to the gratings and the GST is in the amorphous phase as in Fig. 3a and b, a narrow band reflection dip and absorption peak resonant is obtained at $2039 \mathrm{~nm}$ wavelength. If the GST is switched from amorphous to the crystal phase under the same electric field polarization, this resonant wavelength shifts to the longer wavelength (from 2039 to $2143 \mathrm{~nm}$ ), which corresponds to $5 \%$ tuning range. We also note that the absorption peak increases to the perfect absorption level when the GST changes state in addition to a red shift at the resonant wavelength. This can be attributed to the better impedance matching of the structure to the vacuum at the wavelenghth of $2143 \mathrm{~nm}$. Another feature is related to the bandwidth of the resonant wavelength. It is seen that amorphous phase has narrower bandwidth at the resonant wavelength compared to the crystal phase. This difference is caused by the contrast between the optical parameters of two different phases of the GST as seen in Fig. 2b. If the polarization of the electric field vector is parallel to the gratings as in Fig. 3c and d, there exists no such strong resonant behavior between $2 \mu \mathrm{m}$ and $3 \mu \mathrm{m}$ wavelengths in both phases of the GST. While some intensity differences exist at the reflection and absorption spectra of two phases between $2 \mu \mathrm{m}$ and $2.4 \mu \mathrm{m}$, these differences are still less than 0.5 , which may be considered as non-strong resonant situation in comparison to the ones in the vertical electric field polarization. Therefore, we will focus on the results of strong resonant cases.
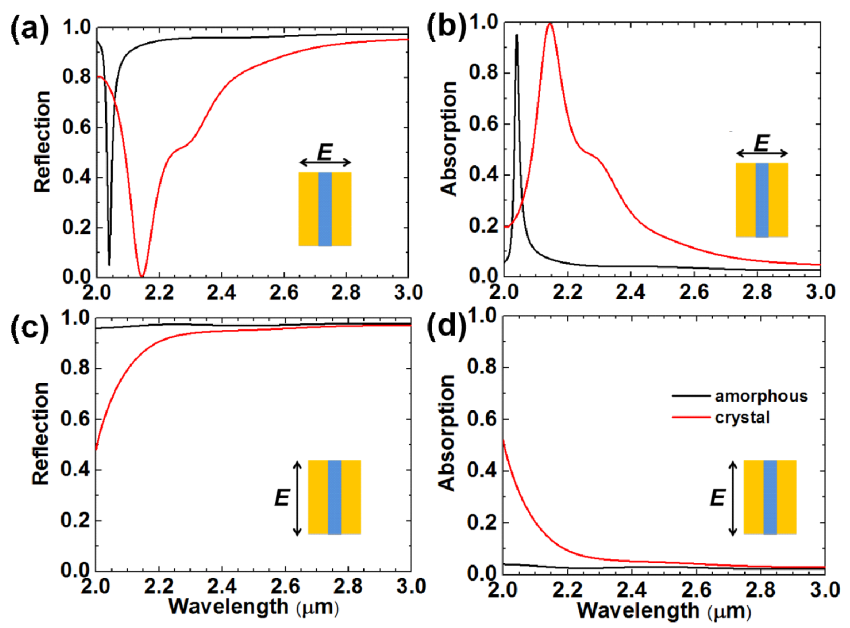

Fig. 3. Simulation results for our design. In all graphs, black curve indicates the amorphous-GST and the red curve indicates the crystal-GST. (a) Reflection vs. wavelength graph when the electric field vector is perpendicular to the gratings. (b) Absorption vs. wavelength graph when the electric field vector is perpendicular to the gratings. (c) Reflection vs. wavelength graph when the electric field vector is parallel to the gratings. (d) Absorption vs. wavelength graph when the electric field vector is parallel to the gratings. 
We can better observe the underlying tunable absorption mechanisms of our design by utilization of the crosssectional $x-y$ plane power absorption map at the resonant wavelengths of amorphous-GST and crystal-GST, which are $2039 \mathrm{~nm}$ and $2143 \mathrm{~nm}$, respectively. Absorbed power $\left(P_{\text {abs }}\right)$ is the divergence of the Poynting vector for non-magnetic materials and can easily be computed using the simple relation $P_{\text {abs }}=\pi c \varepsilon_{0} \varepsilon_{2}|E|^{2} / \lambda$ where $c$ is the speed of the light, $\varepsilon_{0}$ is the free space permittivity, $\varepsilon_{2}$ is the imaginary part of the relative dielectric permittivity, $|E|$ is the absolute magnitude of the total electric field and $\lambda$ is the free-space wavelength $[4,6]$. The results are shown in Fig. 4 where FDTD simulations were performed with an incident electric field vector parallel to the $x$ axis (i.e. vertical polarization). When we look at the two-dimensional colored absorbed power map of our design at the $2039 \mathrm{~nm}$ resonance wavelength (Fig. $4 \mathrm{a}$ and b), $P_{\text {abs }}$ of the amorphous-GST (Fig. 4a) is much more stronger than the crystal-GST case (Fig. 4b). This huge difference is caused by the enhanced power loss inside the top $\mathrm{Au}$ corners and bottom $\mathrm{Au}$ layer as seen in Fig. 4a. At this wavelength, $P_{\text {abs }}$ of the crystal-GST (Fig. 4b) occurs at the relatively small areas of the GST and $\mathrm{Au}$ boundaries and bottom $\mathrm{Au}$, which leads to low absorption compared to the amorphous case.
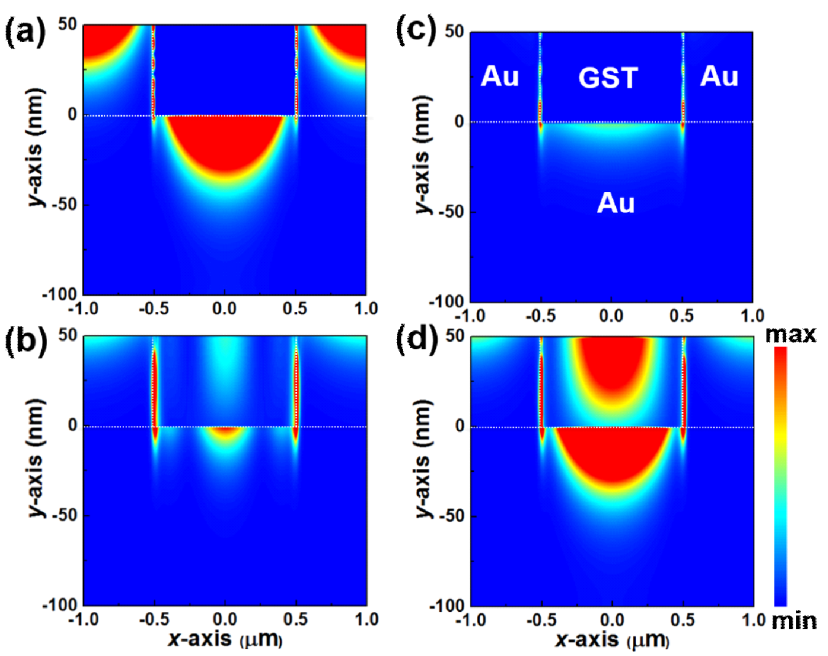

Fig. 4. Cross-sectional $x-y$ plane power absorption $\left(P_{\text {abs }}\right)$ map at $2039 \mathrm{~nm}$ resonant wavelength when GST is in (a) amorphous phase and (b) crystal phase. $P_{\text {abs }}$ map at $2143 \mathrm{~nm}$ resonant wavelength when GST is in (c) amorphous phase and (d) crystal phase.

On the other hand, absorption mechanism at $2143 \mathrm{~nm}$ resonance wavelength (Fig. $4 \mathrm{c}$ and d) is completely reversed with respect to the $2039 \mathrm{~nm}$ resonance wavelength. In this case, $P_{\text {abs }}$ of the crystal-GST (Fig. $4 \mathrm{~d}$ ) is much stronger than the amorphous-GST case (Fig. 4c). This large difference is caused by the enhanced power loss inside the crystal-GST in addition to the bottom Au layer as seen in Fig. 4d. Power absorption at amorphousGST case (Fig. 4c) is negligibly small, which explains why we obtain very low absorption $(\approx 0.05)$ in Fig. $3 \mathrm{~b}$ (amorphous state at $2143 \mathrm{~nm}$ resonance wavelength). Another important observation at this resonant wavelength is the bandwidth enlargement for the crystal GST case. This was attributed to the contrast in the optical material parameters of the GST phases described above. This attribution was further supported and clarified in Fig. 4d. Here, we see enhanced absorption inside the crystal-GST material caused by non-zero and wavelength-dependent $\varepsilon_{2}$ (dashed red line in Fig. 2b).

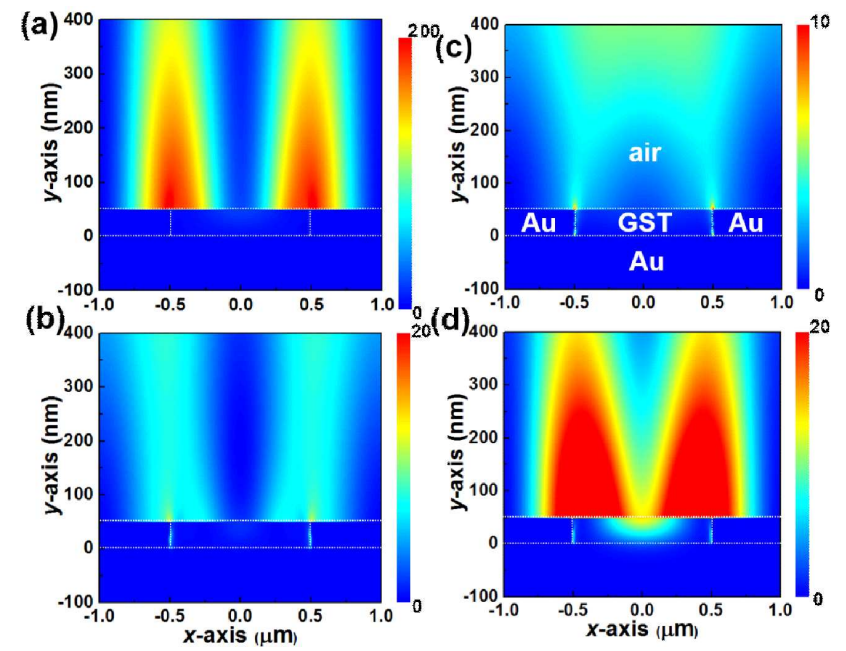

Fig. 5. Cross-sectional $x-y$ plane electric field intensity, $|E|^{2}$, map of our design at $2039 \mathrm{~nm}$ resonant wavelength when GST is in (a) amorphous phase and (b) crystal phase. $|E|^{2}$ map at $2143 \mathrm{~nm}$ resonant wavelength when GST is in (c) amorphous phase and (d) crystal phase.

Different absorption mechanisms described above can be understood in more detail when we look at the electric field intensity $\left(|E|^{2}\right)$ confinement inside the $x-y$ plane cross-sectional structure at the resonant wavelengths of $2039 \mathrm{~nm}$ and $2143 \mathrm{~nm}$. These results were presented in Fig. 5. As expected, $|E|^{2}$ is highly localized along the top edge boundaries between the GST and $\mathrm{Au}$ under resonant conditions of $2039 \mathrm{~nm}$ (Fig. 5a) and $2143 \mathrm{~nm}$ (Fig. 5d). Additionally, the concentration of the field intensity inside the lossy crystal-GST results in further raise of the absorption. On the other hand, there exists negligible electric field intensity penetration for the non-resonant conditions of the same wavelengths (Fig. 5b and c). Therefore, very low absorption values were obtained at these non-resonant wavelengths.

\section{Conclusions}

We have theoretically examined the absorption and reflection properties of the near-infrared light through the nanostructured thin film structures incorporated with the GST as the phase-change material (PCM). Since the optical properties of the GST undergo a substantial change from the amorphous to crystalline phase, 
we have selected GST as the active material. The optical absorption and reflection properties of the designed structure with respect to the geometric and material parameters were systematically investigated using the finite difference time domain computations. It was shown that absorption peak or reflection dip at the resonant wavelengths in the near-infrared region was red shifted from $2039 \mathrm{~nm}$ to $2143 \mathrm{~nm}$ wavelength (which corresponds to $5 \%$ tuning range) by switching the PCM from its amorphous to crystalline states. The distributions of the electric field intensity and the absorbed power at the resonant wavelengths with respect to different phases of the GST were investigated to further explain the physical origin of the absorption. This tunable infrared nanostructured absorber can be fabricated with the existing nanofabrication techniques and can find potential applications such as selective infrared emitters, infrared camouflage, sensors, and photovoltaic devices.

\section{Acknowledgments}

We thank the director and all members of Metamaterials and Nanophotonic Devices Lab of Northwestern University.

\section{References}

[1] N. Landy, S. Sajuyigbe, J.J. Mock, D.R. Smith, W.J. Padilla, Phys. Rev. Lett. 100, 207402 (2008).

[2] H. Tao, N.I. Landy, C.M. Bingham, X. Zhang, R.D. Averitt, W.J. Padilla, Opt. Express 16, 7181 (2008).

[3] H. Kocer, J. Nanophoton. 9, 093597 (2015).

[4] H. Kocer, S. Butun, Z. Li, K. Aydin, Sci. Rep. 5, $8157(2015)$

[5] N. Liu, M. Mesch, T. Weiss, M. Hentschel, H. Giessen, Nano Lett. 10, 2342 (2010).

[6] K. Aydin, V.E. Ferry, R.M. Briggs, H.A. Atwater, Nat. Commun. 2, 517 (2011).
[7] S. Butun, K. Aydin, Opt. Express 22, 19457 (2014).

[8] W. Streyer, S. Law, G. Rooney, T. Jacobs, D. Wasserman, Opt. Express 21, 9113 (2013).

[9] J.J. Talghader, A.S. Gawarikar, R.P. Shea, Light Sci. Appl. 1, e24 (2012).

[10] J.-J. Greffet, R. Carminati, K. Joulain, J.-P. Mulet, S. Mainguy, Y. Chen, Nature 416, 61 (2002).

[11] Y. Avitzour, Y.A. Urzhumov, G. Shvets, Phys Rev. B 79, 045131 (2009).

[12] V.E. Ferry, L.A. Sweatlock, D. Pacifici, H.A. Atwater, Nano Lett 8, 4391 (2008).

[13] H.A. Atwater, A. Polman, Nat. Mater. 9, 205 (2010).

[14] R.A. Pala, J. White, E. Barnard, J. Liu, M.L. Brongersma, Adv. Mater. 21, 3504 (2009).

[15] T. Cao, L. Zhang, R.E. Simpson, M.J. Cryan, J. Opt. Soc. Am. B 30, 1580 (2013).

[16] H.-T. Chen, W.J. Padilla, J.M.O. Zide, A.C. Gossard, A.J. Taylor, R.D. Averitt, Nature 444, 597 (2006).

[17] H.-T. Chen, J.F. O'Hara, A.K. Azad, A.J. Taylor, R.D. Averitt, D.B. Shrekenhamer, W.J. Padilla, Nat. Photon. 2, 295 (2008).

[18] T. Driscoll, S. Palit, M.M. Qazilbash, M. Brehm, F. Keilmann, B.-G. Chae, S.-J. Yun, H.-T. Kim, S.Y. Cho, N.M. Jokerst, D.R. Smith, D.N. Basov, Appl. Phys. Lett. 93, 024101 (2008).

[19] R.L. Voti, M.C. Larciprete, G. Leahu, C. Sibilia, M. Bertolotti, J. Appl. Phys. 112, 034305 (2012).

[20] K. Shportko, S. Kremers, M. Woda, D. Lencer, J. Robertson, M. Wuttig, Nat. Mater. 7, 653 (2008).

[21] T. Cao, C. Wei, R.E. Simpson, L. Zhang, M.J. Cryan, Sci. Rep. 4, 3955 (2014).

[22] Lumerical Solutions, Inc., available at: www. lumerical.com/tcad-products/fdtd/.

[23] E.D. Palik, Handbook of Optical Constants of Solids, Academic Press, New York 1998. 